\title{
MULTISOLITON PHASE SHIFTS IN THE CASE OF A NONZERO REFLECTION COEFFICIENT
}

\author{
Peter SCHUUR \\ Mathematical Institute, Rijksuniversiteit Utrecht, 3508 TA Utrecht, The Netherlands
}

Received 19 April 1984

\begin{abstract}
We study multisoliton solutions of the Korteweg-de Vries equation in the case of a nonzero reflection coefficient. An explicit phase shift formula is derived that clearly displays the nature of the interaction of each soliton with the other ones and with the dispersive wavetrain. In particular, this formula shows that each soliton experiences in addition to the ordinary $N$-soliton phase shift an extra phase shift to the left caused by the collision with the dispersive wavetrain.
\end{abstract}

1. Introduction. We consider the Korteweg-de Vries (KdV) equation $u_{t}-6 u u_{x}+u_{x x x}=0$ with arbitrary initial conditions $u(x, 0)=u_{0}(x)$, which are sufficiently smooth and decay sufficiently rapidly for $|x| \rightarrow \infty$ for the whole of the inverse scattering method to work and to guarantee certain regularity properties of the scattering data, to be stated further on. The long time behaviour of the solution $u(x, t)$ of the $\mathrm{KdV}$ problem has been discussed by numerous authors. The general picture is, that as $t \rightarrow+\infty$ the solution decomposes into $N$ solitons moving to the right and a dispersive wavetrain moving to the left. As $t \rightarrow-\infty$ the arrangement is reversed. The emergence of the $N$ solitons as $t \rightarrow+\infty$ for rather arbitrary classes of initial conditions was demonstrated rigorously in ref. [1] (see also the discussion in ref. [2]). Earlier - but less detailed and not widely known - results in that direction were given in ref. [3]. Further extensions of the asymptotic analysis and improvements of results were recently presented in ref. [4]. In the literature many at tempts were made to calculate the phase shifts of the solitons as they interact both with the other solitons and with the dispersive wavetrain. Many incorrect results were given (cf. refs. [3,5]), until finally the question was settled by Ablowitz and Kodama [6], who presented a correct phase shift formula.

\footnotetext{
"The contents of this paper were presented in the form of a talk and a poster at the Scott Russell Centenary Conference, Edinburgh, 1982.
}

In this paper we rederive this phase shift formula, starting from our asymptotic analysis of the solution given in ref. [4]. We next show how a simple substitution produces a more transparent formula that clearly displays the nature of the interaction of each soliton with the other ones and with the dispersive wavetrain. From our phase shift formula it is evident, that each soliton experiences, in addition to the ordinary $N$. soliton phase shift, an extra phase shift to the left, the so-called continuous phase shift, caused by the collision with the dispersive wavetrain. Thus, the presence of reflection causes a delay in the soliton motion.

The composition of this paper is as follows. In section 2 we briefly discuss the left and right scattering data associated with $u_{0}(x)$ and show how the left scat. tering data can be expressed in terms of the right scattering data in a convenient way. In section 3 we recall a result known from ref. [4], concerning the asymptotic behaviour of $u(x, t)$ as $t \rightarrow+\infty$. By a symmetry argument we derive from this result the asymptotic behaviour of $u(x, t)$ as $t \rightarrow-\infty$. Next, in section 4 , the two asymptotic results are combined to give the Ablowitz-Kodama phase shift formula. The representation of the left normalization coefficient in terms of the right scattering data, which was obtained in section 2, then enables us to write the phase shift formula in a more transparent form. Finally, as an exercise, we calculate in section 5 the continuous phase shifts arising from a sech ${ }^{2}$ initial function. 
2. Scattering data and their properties. For $\operatorname{Im} k$ $\geqslant 0$ we introduce the Jost functions $\psi_{\mathrm{r}}(x, k)$ and $\psi_{Q}(x, k)$, two special solutions of the Schrödinger equation

$\psi_{x x}+\left[k^{2}-u_{0}(x)\right] \psi=0, \quad-\infty<x<\infty$,

determined by

$$
\begin{aligned}
& \psi_{\mathrm{r}}(x, k)=\mathrm{e}^{-\mathrm{i} k x} R(x, k), \\
& \lim _{x \rightarrow-\infty} R(x, k)=1, \quad \lim _{x \rightarrow-\infty} R_{x}(x, k)=0, \\
& \psi_{Q}(x, k)=\mathrm{e}^{\mathrm{i} k x} L(x, k), \\
& \quad \lim _{x \rightarrow+\infty} L(x, k)=1, \quad \lim _{x \rightarrow+\infty} L_{x}(x, k)=0 .
\end{aligned}
$$

We set

$r_{-}(k)=1-\frac{1}{2 \mathrm{i} k} \int_{-\infty}^{\infty} u_{0}(y) R(y, k) \mathrm{d} y$,

$$
k \in \overline{\mathrm{C}}_{+} \backslash\{0\} \text {, }
$$

$r_{+}(k)=\frac{1}{2 \mathrm{i} k} \int_{-\infty}^{\infty} \mathrm{e}^{-2 \mathrm{i} k y_{u}} u_{0}(y) R(y, k) \mathrm{d} y$,

$$
k \in \mathrm{R} \backslash\{0\} \text {, }
$$

$l_{+}(k)=1-\frac{1}{2 \mathrm{i} k} \int_{-\infty}^{\infty} u_{0}(y) L(y, k) \mathrm{d} y$,

$$
k \in \overline{\mathrm{C}}_{+} \backslash\{0\} \text {, }
$$

$l_{-}(k)=\frac{1}{2 \mathrm{i} k} \int_{-\infty}^{\infty} \mathrm{e}^{2 \mathrm{i} k y} u_{0}(y) L(y, k) \mathrm{d} y$,

$$
k \in \mathrm{R} \backslash\{0\} .
$$

Note that $r_{-}(k)=l_{+}(k)$, whereas $r_{+}(k)=-l_{-}(-k)$. It is well known [2], that $r_{-}(k)$ is analytic on $C_{+}$with at most finitely many zeros, all simple and on the imaginary axis. Let us denote them by i $\kappa_{m}, m=1,2$, ..., $N$ and order

$k_{1}>k_{2}>\ldots>k_{N}>0$.

Bearing in mind that $\psi_{\ell}\left(x, \mathrm{i} \kappa_{m}\right)$ and $\psi_{\mathrm{r}}\left(x, \mathrm{i} \kappa_{m}\right)$ are both real valued and square integrable, we introduce

$C_{m}^{\mathrm{r}}=\left[\int_{-\infty}^{\infty} \psi_{l}^{2}\left(x, i \kappa_{m}\right) \mathrm{d} x\right]^{-1 / 2}$, the right normalization coefficients,

$C_{m}^{\ell}=\left[\int_{-\infty}^{\infty} \psi_{\mathrm{r}}^{2}\left(x, \mathrm{i} \kappa_{m}\right) \mathrm{d} x\right]^{-1 / 2}$,

the left normalization coefficients.

Furthermore, we introduce the following quantities for $k \in R \backslash\{0\}$

$a_{\mathrm{r}}=r_{-}^{-1}$,

the right transmission coefficient,

$a_{\ell}=l_{+}^{-1}$,

the left transmission coefficient,

$b_{\mathrm{r}}=r_{+} r_{-}^{-1}$,

the right reflection coefficient,

$b_{Q}=l_{-} l_{+}^{-1}$,

the left reflection coefficient.

Assuming that $u_{0}(x)$ decays sufficiently rapidly (see ref. [2]) we can extend $a_{\mathrm{r}}, a_{\ell}, b_{\mathrm{r}}, b_{\ell}$ in a natural way to continuous functions on all of $R$. We shall call the aggregate of quantities $\left\{a_{\mathrm{r}}(k), b_{\mathrm{r}}(k), k_{m}, c_{m}^{\mathrm{r}}\right\}$ the right scattering data of the potential $u_{0}$. Similarly we refer to $\left\{a_{\ell}(k), b_{Q}(k), \kappa_{m}, c_{m}^{\ell}\right\}$ as the left scattering data associated with $u_{0}$. We claim that $a_{\ell}, b_{\ell}$ and $c_{m}^{\ell}$ can be expressed in terms of the right scattering data in the following way

$$
\begin{aligned}
& a_{\ell}(k)=a_{\mathrm{r}}(k), \quad b_{\ell}(k)=-\frac{a_{\mathrm{r}}(k)}{a_{\mathrm{r}}(-k)} b_{\mathrm{r}}(-k), \\
& c_{m}^{\ell}=\left(c_{m}^{\mathrm{r}}\right)^{-1} 2 \kappa_{m} \exp \left(\frac{\kappa_{m}}{\pi} \int_{0}^{\infty} \frac{\log \left(1-\left|b_{\mathrm{r}}(k)\right|^{2}\right)}{k^{2}+\kappa_{m}^{2}} \mathrm{~d} k\right) \\
& \quad \times \prod_{\substack{p=1 \\
p \neq m}}^{N}\left|\frac{\kappa_{m}+\kappa_{p}}{\kappa_{m}-\kappa_{p}}\right| .
\end{aligned}
$$

Indeed, the relations (a) are obvious. To derive (7b) we combine certain familiar facts from refs. $[2,7]$. Firstly, from ref. [2], p. 110 we know

$\psi_{\mathrm{I}}\left(x, \mathrm{i} \kappa_{m}\right)=\alpha_{m} \psi_{\mathrm{Q}}\left(x, \mathrm{i} \kappa_{m}\right)$, with $\alpha_{m} \in \mathrm{R} \backslash\{0\}$.

Hence, by (5)

$c_{m}^{\mathrm{r}}=\left|\alpha_{m}\right| c_{m}^{\ell}$.

Next, by ref. [2], (4.3.18) one has 


$$
\begin{aligned}
& \left.\frac{\mathrm{d} r_{-}}{\mathrm{d} k}\right|_{k=\mathrm{i} \kappa_{m}}=\frac{1}{\mathrm{i} \alpha_{m}} \int_{-\infty}^{\infty} \psi_{\mathrm{r}}^{2}\left(x, \mathrm{i} \kappa_{m}\right) \mathrm{d} x \\
& =\left(\mathrm{i} \alpha_{m}\right)^{-1}\left(c_{m}^{\ell}\right)^{-2} .
\end{aligned}
$$

Eliminating $\alpha_{m}$ from (9) and (10) we find

$c_{m}^{\mathrm{r}} c_{m}^{\ell}\left|\frac{\mathrm{d} r_{-}}{\mathrm{d} k}\right|_{k=\mathrm{i} \kappa_{m}} \mid=1$.

Lastly, from ref. [7], p. 154 we obtain the representation

$$
\begin{aligned}
& r_{-}(k)=\exp \left(\frac{1}{2 \pi \mathrm{i}} \int_{-\infty}^{\infty} \frac{\log \left(1-\left|b_{\mathrm{r}}(\omega)\right|^{2}\right)}{k-\omega} \mathrm{d} \omega\right) \\
& \times \prod_{p=1}^{N} \frac{k-\mathrm{i} \kappa_{p}}{k+\mathrm{i} \kappa_{p}}, \quad \operatorname{Im} k>0 .
\end{aligned}
$$

Consequently

$$
\begin{aligned}
& \left|\frac{\mathrm{d} r_{-}}{\mathrm{d} k}\right|_{k=\mathrm{i} \kappa_{m}} \mid \\
& \quad=\frac{1}{2 \kappa_{m}} \exp \left(-\frac{\kappa_{m}}{\pi} \int_{0}^{\infty} \frac{\log \left(1-\left|b_{\mathrm{r}}(k)\right|^{2}\right)}{k^{2}+\kappa_{m}^{2}} \mathrm{~d} k\right) \\
& \quad \times \prod_{\substack{p=1 \\
p \neq m}}^{N}\left|\frac{\kappa_{m}-\kappa_{p}}{\kappa_{m}+\kappa_{p}}\right|,
\end{aligned}
$$

where we have used that $b_{\mathrm{r}}^{*}(k)=b_{\mathrm{r}}(-k)$. Combining (11) and (13) we arrive at the desired formula (7b).

\section{Forward and backward asymptotics. Once the} right scattering data of $u_{0}(x)$ are known, the solution $u(x, t)$ of the forward $\mathrm{KdV}$ problem

$u_{t}-6 u u_{x}+u_{x x x}=0, \quad t>0$,

$u(x, 0)=u_{0}(x)$,

can in principle be computed by the inverse scattering method [2]. Concerning the asymptotic behaviour of the solution we have obtained the following result in ref. [4]:

Lemma 1. There is an integer $n^{+} \geqslant 2$, such that $b_{\mathrm{r}}(k)$ is of class $\mathrm{C}^{3 n^{+}-4}(\mathrm{R})$ and all derivatives $b_{\mathrm{r}}^{(j)}(k)$, $j=0,1, \ldots, 3 n^{+}-4$ satisfy

$b_{\mathrm{r}}^{(j)}(k)=\mathrm{O}\left(k^{-x_{n^{+}}}\right), \quad k \rightarrow \pm \infty$, where $\chi_{2}=1$ and $\chi_{\rho}=0$ for $\rho=3,4, \ldots$. Put

$\Delta(\rho)=\frac{1}{3}-1 / 2\left(3 \rho-5+\chi_{\rho}\right), \quad \rho=2,3, \ldots$.

Then for any choice of the constant $0 \leqslant \delta<\Delta\left(n^{+}\right)$ one has

$$
\begin{aligned}
& \lim _{t \rightarrow \infty} \sup _{x \geqslant-t^{\delta}} \mid u(x, t) \\
& \quad-\sum_{m=1}^{N}\left\{-2 \kappa_{m}^{2} \operatorname{sech}^{2}\left[\kappa_{m}\left(x-x_{m}^{+}-4 \kappa_{m}^{2} t\right)\right]\right\} \mid=0,
\end{aligned}
$$

where

$x_{m}^{+}=\frac{1}{2 \kappa_{m}} \log \left[\frac{\left[c_{m}^{\Gamma}\right]^{2}}{2 \kappa_{m}} \prod_{p=1}^{m-1}\left(\frac{\kappa_{p}-\kappa_{m}}{\kappa_{p}+\kappa_{m}}\right)^{2}\right]$.

Let us now consider the backward $\mathrm{KdV}$ problem, starting from the same initial function $u_{0}(x)$, i.e.

$u_{t}-6 u u_{x}+u_{x x x}=0, \quad t<0$,

$u(x, 0)=u_{0}(x)$.

Clearly, if $u(x, t)$ satisfies (19), then $w(x, t)=u(-x$, $-t)$ satisfies

$w_{t}-6 w w_{x}+w_{x x x}=0, t>0$,

$w(x, 0)=u_{0}(-x)$,

so that $w(x, t)$ satisfies the forward $\mathrm{KdV}$ problem with initial function $u_{0}(-x)$. To solve (19) it is therefore sufficient to determine the right scattering data associated with $u_{0}(-x)$ and apply the inverse scattering meth. od to $(20)$. However, it is readily verified that the right scattering data associated with $u_{0}(-x)$ are equal to the left scattering data associated with $u_{0}(x)$, which were studied in the previous section. Thus, to find the asymptotic behaviour of the solution $u(x, t)$ of (19) for $t \rightarrow-\infty$ we merely apply lemma 1 to problem (20) and perform the transcription $u(x, t)=w(-x,-t)$. This yields (in the notation introduced in lemma 1 ):

Lemma 2. Assume that: There is an integer $n^{-} \geqslant 2$ such that $b_{Q} \in \mathrm{C}^{3 n^{-}-.4}(\mathrm{R})$ and all derivatives $b_{Q}^{(j)}(k)$, $j=0,1, \ldots, 3 n^{-}-4$ satisfy

$b_{\ell}^{(j)}(k)=O\left(k^{-x_{n^{-}}}\right), \quad k \rightarrow \pm \infty$.

Then for any choice of the constant $0 \leqslant \delta<\Delta\left(n^{-}\right)$ one has 


$$
\begin{aligned}
\lim _{t \rightarrow-\infty} \sup _{x \in|t|^{\delta}} \mid u(x, t) \\
\quad-\sum_{m=1}^{N}\left(-2 \kappa_{m}^{2} \operatorname{sech}^{2}\left[\kappa_{m}\left(x-x_{m}^{-}-4 \kappa_{m}^{2} t\right)\right]\right) \mid=0
\end{aligned}
$$

where

$x_{m}^{-}=-\frac{1}{2 \kappa_{m}} \log \left[\frac{\left[c_{m}^{\ell}\right]^{2}}{2 \kappa_{m}} \prod_{p=1}^{m-1}\left(\frac{\kappa_{p}-\kappa_{m}}{\kappa_{p}+\kappa_{m}}\right)^{2}\right]$.

Note that $b_{\ell}$ and $b_{\mathrm{r}}$ may be quite different in nature, which affects the convergence results (17) and (22). For instance, if $u_{0}(x)$ has support on a left half-line, then (17) will hold for values of $\delta$ close to $\frac{1}{3}$ whereas (22) may only be true for $\delta$ close to 0 .

\section{An explicit phase shift formula. Let us assume} that $b_{\mathrm{r}}$ and $b_{\ell}$ satisfy the conditions (15) and (21). Then the convergence results (17) and (22) display clearly how the solution $u(x, t)$ of the $\mathrm{KdV}$ equation evolving from $u(x, 0)=u_{0}(x)$ splits up into $N$ solitons as $t \rightarrow \pm \infty$. In particular, we find for the $m$ th soliton the following phase shift

$$
\begin{aligned}
S_{m} & =x_{m}^{+}-x_{m}^{-} \\
& =\frac{1}{2 \kappa_{m}} \log \left[\left(\frac{c_{m}^{\mathrm{r}} c_{m}^{\ell}}{2 \kappa_{m}}\right)^{2} \prod_{p=1}^{m-1}\left(\frac{\kappa_{m}-\kappa_{p}}{\kappa_{m}+\kappa_{p}}\right)^{4}\right] .
\end{aligned}
$$

This formula was first derived by Ablowitz and Segur [8] for the $N=1$ case and by Ablowitz and Kodama [6] for the $N>1$ case (see also the discussion in ref. [9]).

It is a remarkable fact that the formulas (23) and (24) become more transparent if one inserts the representation (7b). Summarizing, this leads to

$x_{m}^{+}=\frac{1}{2 \kappa_{m}} \log \left[\frac{\left[c_{m}^{\mathrm{r}}\right]^{2}}{2 \kappa_{m}}\right]+\frac{1}{\kappa_{m}} \sum_{p=1}^{m-1} \log \left(\frac{\kappa_{p}-\kappa_{m}}{\kappa_{p}+\kappa_{m}}\right)$,

$x_{m}^{-}=\frac{1}{2 \kappa_{m}} \log \left[\frac{\left[c_{m}^{\mathrm{r}}\right]^{2}}{2 \kappa_{m}}\right]+\frac{1}{\kappa_{m}} \sum_{p=m+1}^{N} \log \left(\frac{\kappa_{m}-\kappa_{p}}{\kappa_{m}+\kappa_{p}}\right)$

$$
-\frac{1}{\pi} \int_{0}^{\infty} \frac{\log \left(1-\left|b_{\mathrm{r}}(k)\right|^{2}\right)}{k^{2}+\kappa_{m}^{2}} \mathrm{~d} k \text {, }
$$

$S_{m}=S_{m}^{\mathrm{d}}+S_{m}^{\mathrm{c}}$,

$$
\begin{aligned}
S_{m}^{\mathrm{d}} & =\frac{1}{\kappa_{m}} \sum_{p=1}^{m-1} \log \left(\frac{\kappa_{p}-\kappa_{m}}{\kappa_{p}+\kappa_{m}}\right) \\
& -\frac{1}{\kappa_{m}} \sum_{p=m+1}^{N} \log \left(\frac{\kappa_{m}-\kappa_{p}}{\kappa_{m}+\kappa_{p}}\right),
\end{aligned}
$$

$S_{m}^{c}=\frac{1}{\pi} \int_{0}^{\infty} \frac{\log \left(1-\left|b_{\mathrm{r}}(k)\right|^{2}\right)}{k^{2}+k_{m}^{2}} \mathrm{~d} k$.

In $S_{m}^{\mathrm{d}}$ we recognize the pure $N$-soliton phase shift (caused by pairwise interaction of the $m$ th soliton with the other ones). The quantity $S_{m}^{\mathrm{c}}$ (which is negative for nonzero $b_{\mathrm{I}}$ ) can be seen as the shift caused by the interaction of the $m$ th soliton with the dispersive wavetrain. Note that the phase shift $S_{m}$ is completely determined by the bound states $\kappa_{p}$ and the right reflection coefficient $b_{\mathrm{r}}$. For nonzero $b_{\mathrm{r}}$ we obviously have

$0>S_{1}^{\mathrm{c}}>S_{2}^{\mathrm{c}}>\ldots>S_{N}^{\mathrm{c}}$.

Thus, the collision with the dispersive wavetrain causes a delay in the motion of the solitons and the effect is most heavily felt by the smallest one, corresponding to $\kappa_{N}$. Using the formula (see ref. [9])

$$
\begin{aligned}
& \int_{-\infty}^{\infty} u_{0}(x) \mathrm{d} x=-\frac{2}{\pi} \int_{0}^{\infty} \log \left(1-\left|b_{\mathrm{r}}(k)\right|^{2}\right) \mathrm{d} k \\
& -4 \sum_{p=1}^{N} \kappa_{p},
\end{aligned}
$$

we obtain for the continuous phase shift $S_{m}^{\mathrm{c}}$ the following estimate in terms of the initial function $u_{0}(x)$ and the bound states $\kappa_{p}$ :

$$
\left|S_{m}^{\mathrm{c}}\right| \leqslant \frac{1}{2 \kappa_{m}^{2}}\left(\int_{-\infty}^{\infty} u_{0}(x) \mathrm{d} x+4 \sum_{p=1}^{N} \kappa_{p}\right) .
$$

In estimating the size of $S_{m}^{\mathrm{c}}$ one has to distinguish two cases, the "generic case" and the "exceptional case" (see refs. $[7,10]$ ). In the generic case, the Jost functions $\psi_{\mathrm{r}}(x, 0)$ and $\psi_{Q}(x, 0)$ are linearly independent, whereas in the exceptional case they are not. In the exceptional case one has

$$
B=\sup _{k \in \mathrm{R}}\left|b_{\mathrm{r}}(k)\right|<1 \text {, }
$$

whence 
$\left|S_{m}^{\mathfrak{c}}\right| \leqslant-\left(1 / 2 \kappa_{m}\right) \log \left(1-B^{2}\right)$.

In the generic case there is an $\alpha \neq 0$ with

$b_{\mathrm{r}}(k)=-1+\alpha k+\mathrm{o}(k)$, as $k \rightarrow 0$,

so that in the integral defining $S_{m}^{\mathrm{c}}$ the contribution of $k=0$ becomes important. In particular, fixing $\left|b_{\mathrm{r}}\right|$, we find for $\kappa_{m} \downarrow 0$

$S_{m}^{c} \sim\left(1 / 2 \kappa_{m}\right) \log \left(1-\left|b_{r}(0)\right|^{2}\right)$,

in the exceptional case,

$S_{m}^{\mathrm{c}} \sim\left(1 / \kappa_{m}\right) \log \kappa_{m}$,

in the generic case.

Clearly, in general the sizes of $S_{m}^{\mathrm{c}}$ and $S_{m}^{\mathrm{d}}$ are incomparable. On the other hand one can easily construct examples in which one of the two dominates. For instance, consider a generic case with two bound states $\kappa_{1}$ and $\kappa_{2}=\frac{1}{2} \kappa_{1}$.

Then, for fixed $\left|b_{r}\right|$, the discrete phase shifts $S_{m}^{\mathrm{d}}$ dominate for $\kappa_{1} \rightarrow+\infty$, whereas the continuous phase shifts $S_{m}^{\mathrm{c}}$ dominate for $\kappa_{1} \downarrow 0$; in the $\kappa_{1} \downarrow 0$ case the familiar picture of a KdV soliton overtaking a smaller one, where the smaller one is shifted to the left and the larger to the right, changes, since now both are shifted to the left.

\section{An example: the continuous phase shifts arising} from a sech ${ }^{2}$ initial function. To illustrate the previous discussion let us compute the continuous phase shifts arising from the initial function

$u_{0}(x)=-\lambda(\lambda+1) \operatorname{sech}^{2} x, \quad \lambda>0$.

From ref. [11] we find

$a_{\mathrm{r}}(k)=\frac{\Gamma(a) \Gamma(b)}{\Gamma(c) \Gamma(a+b-c)}$,

$b_{\mathbf{r}}(k)=\frac{\Gamma(c-a-b) \Gamma(a) \Gamma(b)}{\Gamma(c-a) \Gamma(c-b) \Gamma(a+b-c)}$,

with

$a=1+\lambda-\mathrm{i} k, \quad b=-\lambda-\mathrm{i} k, \quad c=1-\mathrm{i} k$,

where $\Gamma$ denotes the gamma function (ref. [12],p.

253). Clearly, $a_{\mathrm{r}}$ is analytic on $\mathrm{C}_{+} \backslash\left\{\kappa_{1}, \kappa_{2}, \ldots, \kappa_{N}\right\}$ with simple poles at the bound states $\kappa_{1}, \kappa_{2}, \ldots, \kappa_{N}$. Here $N \geqslant 1$ is the unique integer such that $N-1<\lambda$ $\leqslant N$ and the $\kappa_{p}$ are given by $\kappa_{p}=1+\lambda-p, \quad p=1,2, \ldots, N$.

Note, that $u_{0}(x)$ is reflectionless (i.e. $b_{\tau} \equiv 0$ ) if and only if $\lambda=1,2, \ldots$, in which case $N=\lambda$. For the other values of $\lambda$ we find that $b_{\mathrm{f}}(0)=-1$ so that we are in the generic case. To compute the continuous phase shifts $S_{m}^{\mathrm{c}}$ we notice that by (35)

$r_{-}(\mathrm{i} \nu)=\frac{\Gamma(\nu) \Gamma(1+\nu)}{\Gamma(1+\lambda+\nu) \Gamma(\nu-\lambda)}, \quad \nu>0$.

On the other hand, by (12)

$$
\begin{aligned}
& r_{-}(\mathrm{i} \nu)=\exp \left(-\frac{\nu}{\pi} \int_{0}^{\infty} \frac{\log \left(1-\left|b_{\mathrm{r}}(k)\right|^{2}\right)}{k^{2}+\nu^{2}} \mathrm{~d} k\right) \\
& \times \prod_{p=1}^{N} \frac{\nu-\kappa_{p}}{\nu+\kappa_{p}}, \quad \nu>0 .
\end{aligned}
$$

Equating both expressions we obtain, after repeated use of the recurrence formula $\Gamma(z+1)=z \Gamma(z)$, the following identity

$$
\begin{aligned}
& \frac{1}{\pi} \int_{0}^{\infty} \frac{\log \left(1-\left|b_{\mathrm{r}}(k)\right|^{2}\right)}{k^{2}+\nu^{2}} \mathrm{~d} k \\
& \quad=-\frac{1}{\nu} \log \left(\frac{\Gamma(\nu) \Gamma(1+\nu)}{\Gamma(1+\nu+\lambda-N) \Gamma(\nu-\lambda+N)}\right) \\
& \quad=-\frac{1}{\nu} \log \left(\frac{B(\nu, 1+\nu)}{B(1+\nu+\lambda-N, \nu-\lambda+N)}\right),
\end{aligned}
$$

where $B$ refers to the beta function (ref. [12], p. 258). Finally, combining (36) and (39), we find that the continuous phase shifts $S_{m}^{c}$ are given by

$$
\begin{gathered}
S_{m}^{\mathfrak{c}}=-\frac{1}{1+\lambda-m} \log \left(\frac{B(2-m+\lambda, 1-m+\lambda)}{B(2-m+2 \lambda-N, 1-m+N)}\right) \\
m=1,2, \ldots, N .
\end{gathered}
$$

However, to get an idea of the magnitude of $S_{m}^{\mathrm{c}}$ it is much simpler to employ the estimate (29) which gives us immediately

$\left|S_{m}^{\mathrm{c}}\right| \leqslant \frac{(N-\lambda)(1+\lambda-N)}{(1+\lambda-m)^{2}}$.

I would like to thank F. van der Blij, W. Eckhaus, A. van Harten and Y. Kodama for helpful conversations. 


\section{References}

[1] W. Eckhaus and P. Schuur, Math. Meth. Appl. Sci. 5 (1983) 97.

[2] W. Eckhaus and A. van Harten, The inverse scattering transformation and the theory of solitons. NorthHolland Mathematics Studies 50 (North-Holland, Amsterdam, 1981).

[3] S. Tanaka, Korteweg-de Vries equation; Asymptotic behavior of solutions. Publ. R.I.M.S. Kyoto Univ. 10 (1975) pp. 367-379.

[4] P. Schuur, Asymptotic estimates of solutions of the Kortweg-de Vries equation on right half lines slowly moving to the left, preprint 330 Mathematical Institute Utrecht (1984).
[5] V.E. Zakharov, Sov. Phys. JETP 33 (1971) 538.

[6] M.J. Ablowitz and Y. Kodama, Stud. Appl. Math. 66 (1982) 159

[7] P. Deift and E. Trubowitz, Comm. Pure Appl. Math. 32 (1979) 121.

[8] M.J. Ablowitz and H. Segur, Stud. Appl. Math. 57 (1977) 13.

[9] M.J. Ablowitz and H. Segur, Solitons and the inverse scattering transform (SIAM, Philadelphia, 1981).

[10] A. Cohen, Arch. Rat. Mech. Anal. 71 (1979) 143.

[11] G.L. Lamb Jr., Elements of soliton theory (WileyInterscience, New York, 1980).

[12] M. Abramowitz and I.A. Stegun, Handbook of mathematical functions, National Bureau of Standards Applied Mathematics Series, No. 55, US Department of Commerce (1964). 